\title{
NON INVASIVE TREATMENT OF UTERINE FIBROIDS USING MR GUIDED FOCUSED ULTRASOUND WITH FOLLOW UP - A CASE REPORT
}

Anindita Mishra ${ }^{1}$, N. Vinay ${ }^{2}$

\section{HOW TO CITE THIS ARTICLE:}

Anindita Mishra, N Vinay. "Non invasive treatment of uterine fibroids using MR guided focused ultrasound with follow up - a case report". Journal of Evolution of Medical and Dental Sciences 2013; Vol. 2, Issue 41, October 14; Page: 7903-7908.

ABSTRACT: Uterine leiomyomas are the most common benign tumors in women of child bearing age. They can cause symptoms including dysmenorrhoea, menorrhagia urinary symptoms and infertility. MR guided focused ultrasound is a non-invasive treatment approach for symptomatic uterine fibroids. We present a case of uterine fibroids treated with MRgFUS showing good symptomatic relief and lesion regression at six months follow up.

INTRODUCTION: Uterine leiomyomas are common benign gynecological disorders that affect women in reproductive age group causing a significant impact on a woman's health and quality of life. Hysterectomy which eliminates the source of underlying symptoms is a common approach to treating leiomyomas. Less invasive approaches such as myomectomy, uterine artery embolization and hormonal therapy have been shown to alleviate symptoms. MRgFUS is the only truly noninvasive technique for treatment of these conditions. In clinical trials MRgFUS resulted in significant relief of symptoms due to fibroids and focal adenomyosis. Our case report demonstrates the successful use of MRgFUS to treat symptomatic uterine fibroids.

CASE: This patient was a 49 year old multiparous woman with complaints of menorrhagia and metrorrhagia. Her symptom severity score (on the score of 0-100 Scale of the UFS - QoL, questionnaire ${ }^{1}$ was 48 points. .MRI showed an anterior wall and fundal intramural fibroid measuring $5.6 \times 5.1 \times 5.7 \mathrm{cms}$ (volume 76cc). The fibroid was hypointense to myometrium on T2W images (fig. 1 A\&B). Post-gadolinium contrast images showed moderate heterogenous enhancement (fig. 1C).

After obtaining written consent from the patient, treatment was performed using the ExAblate 2000 system (Insightec, Hafia, Israel) and SIGNA HDxt 1.5T MRI (GE Healthcare, Milwaukee, U.S.A)

On the day of treatment patient was administered conscious sedation (one ampoule of Fentanyl) to reduce motion during the procedure. A urinary catheter was inserted and bowels were mitigated using a rectal balloon (fig. 1 D). The fibroid was treated using a total number of 28 sonication spots and treatment duration was 50 minutes (from first to last sonication) (fig. $1 \mathrm{E}-\mathrm{H}$ ) Mean energy was 2045 joules and frequency of sonication waves was $1.15 \mathrm{M}$. Temperature achieved was in the range of $60-85^{\circ} \mathrm{C}$. Post treatment contrast enhanced SPGR sequences were acquired which showed a non-perfused volume of 72cc (fig. 1I \&J) achieving an NPV ratio of 95\%. Immediate post treatment period was uneventful and patient was discharged after removal of urinary catheter and rectal balloon. The patient was comfortable and returned to her daily routine the next day. On follow up after 6months there was significant alleviation of symptoms and her symptom severity score was reduced to 20. Follow up MRI showed a decrease in dimensions of the fibroid in all three planes. It measured $4.5 \times 4.2 \times 4.7 \mathrm{cms}$ (fig. $1 \mathrm{~K} \& \mathrm{~L}$ ). 
DISCUSSION: Uterine fibroids are common benign neoplasms of unknown etiology. The symptoms include menorrhagia, dysmenorrhea, back pain, urinary symptoms and infertility. ${ }^{2} \mathrm{On}$ ultrasound fibroids appear as well defined echogenic or hypoechoic solid masses with a degree of posterior acoustic shadowing even in non-calcified fibroids. MRI is the most sensitive method for accurately diagnosing uterine fibroids. On MRI typically non degenerated fibroids are well-defined masses of low signal intensity as compared to the myometrium on $\mathrm{T} 2 \mathrm{~W}$ images and isointense to myometrium on T1W images. Degenerated fibroid show complex appearance with heterogeneous or high signal on T2W images.

Definitive treatment for fibroids has traditionally been hysterectomy but increasingly women are not prepared to undergo such an invasive procedure for a benign and usually selflimiting condition. ${ }^{3}$ For women who wish to preserve the uterus and retain their reproductive functions alternative treatments including, myomectomy, and uterine artery embolization (UAE) are now available. ${ }^{4}$ Myomectomy done to remove fibroids is a major abdominal surgery. The potential complications include blood loss, infection and adhesions which in the long run may impair fertility. Uterine artery embolization in the recent years has emerged as a less invasive treatment option for myomas but the tradeoffs include a higher percentage of complications (miscarriage, preterm delivery, intrauterine growth retardation, abnormal placentation, malpresentation and postpartum haemorrhage, ${ }^{5}$ suggesting that further research is needed before the procedure is widely accepted. Since all surgeries have potential complications (anesthesia and post op infection) and post op sequelae such as adhesions, patients may opt for less invasive alternatives which will allow them to resume their normal activities faster. ${ }^{4}$

A recent non-invasive treatment option for fibroids is MR guided focal ultrasound 3 (MRgFUS) is a non-invasive treatment option for symptomatic uterine fibroids. Several clinical trials demonstrate that MRgFUS is a safe and effective treatment for uterine fibroids. ${ }^{2,3}$, $5 \mathrm{MR}$ guided focused ultrasound has a considerable advantage over other techniques as it is completely noninvasive and can be carried out as a day care procedure. ${ }^{3 \mathrm{MR}}$ guided focused ultrasound is the only totally noninvasive surgical approved method for treatment of uterine fibroids. ${ }^{2}$ In clinical trials MRgFUS resulted in significant relief of symptoms due to uterine fibroids2This procedure does not involve any surgery thus avoiding scar formation. For women who wish to preserve their reproductive functions and retain the possibility of future pregnancy this procedure is a safe alternative to various more invasive techniques. ${ }^{2,6}$

Magnetic resonance focused ultrasound surgery is currently used in our center for treatment of symptomatic uterine fibroids. It utilizes precisely focused ultrasound waves to generate and maintain high temperatures within the targeted tissue thus inducing protein denaturation and coagulative necrosis. ${ }^{7}$

The treatment incorporates a 1.5TMRI scanner (Signa HD, GE Healthcare, Milwaukee, WI, USA) along with a focused ultrasound system (ExAblate 2000, Insightec, Haifa, Israel). The operator uses the integrated system to deliver accurate energy pulses (termed sonications) to a location identified on anatomical T2 weighted MRI images. The heat generated during the course of the treatment is monitored using images real time. At the end of the treatment, the results are evaluated by the non-perfused areas on contrast enhanced SPGR images. These areas are summed to create a volume termed non-perfused volume (NPV). The ratio of the NPV to the volume of the fibroid or the 
NPV ratio has been correlated with the efficacy of treatment and shrinkage in size of the fibroid on follow up studies. ${ }^{8}$

MRgFUS is a day care procedure with low incidence of complication. . A number of reports have been published showing the increased probability of pregnancy following MRgFUS. ${ }^{9}$ But not all patients are considered suitable for the procedure. Potential candidates are screened with pelvic MRI to determine whether the meet the patient selection guidelines. The safe and effective use of MRgFUS is affected by fibroid location and type, position relative to adjacent anatomical structures such as bowels and presence of coexistent pelvic disease. Additionally successful outcomes with MRgFUS have been correlated with the volume of fibroid ablated during the procedure (NPV). ${ }^{2}$

An important factor to be considered while selecting suitable patients is the imaging characteristics of the fibroid. The signal intensity of the fibroid as compared to the surrounding myometrium on T2WI images is of significance in assessing the outcome of treatment. The capacity of a fibroid to absorb heat which is based on the signal intensity of the fibroid determines the effects of thermal ablation of the fibroid tissue. Previous reports suggest the average non-perfused volume (NPV) achieved is higher with iso or hypo-intense fibroids as compared to hyperintense fibroids. ${ }^{8}$ ${ }^{10}$ In this case the fibroid was hypointense as compared to normal myometrium and an NPV of 95\% was achieved in the first case and an NPV of $90 \%$ and $85 \%$ were achieved for the fundal and posterior wall fibroid respectively in the second case. This correlates with the significant shrinkage in size and alleviation of symptoms at six months follow up.

\section{REFERENCES:}

1. Spies JB, Coyne K, Guaou N, Boyle D, Skyrnarz-Murphy K, Gonzalves SM. The UFS-QOL, a new disease specific symptom and health related quality of life questionnaire for leiomyomata. Obstetric and Gyanecology.2002;99(2):290-300.PMID:1181451

2. Yoon S-W, Lee C, Cha SH et al. Patient selection guidelines in MR guided focused ultrasound surgery for uterine fibroids: A pictorial guide to relevant findings in screening pelvic MRI. European Radiology.2008; 18(12):2997-3006 PMID:18618119

3. A Chapman, G. TerHaar et al. Thermal ablation of uterine fibroid using MR-guided focused ultrasound-A truly invasive treatment modality. European Radiology.2007; 17(10):25052511.PMID 1935676.

4. Somigliana E, Vercellini P, Daguati R, Pasin R, De Giorgi O, Crosignani PG. Fibroids and female reproduction: a critical analysis of the evidence. Human Reproductive Update 2007;13:46576.PMID 17584819

5. Walker WJ, McDowel l SJ. Pregnancy after uterine artery embolization for leiomyomata: a series of 56 completed pregnancies. Am J Obstet Gynecol 2006; 195:1266-1271 PMID:16796984

6. Rabinovici J, Inbar Y, Revel A et al. Clinical improvement and shrinkage of uterine fibroids after thermal ablation by magnetic resonance-guided focused ultrasound surgery. Ultrasound in Obstetrics and Gynecology.2007; 3(5):771-777.PMID:17899577.

7. Fennessy FM, Tempany CM. A review of magnetic resonance image - guided focused ultrasound surgery in uterine fibroids .Topics in Magnetic Resonance Imaging.2006;17(3):173-179.PMID 17414074 
8. Funaki K, Fukunashi H, Funaki T, Sawada K, Kaji Y, Maruo T. Magnetic resonance-guided focused ultrasound surgery for uterine fibroids: relationship between the therapeutic effects and signal intensity of preexisting T2-weighted magnetic resonance images. AM J Obstet Gynecol 2007; 196:184.e1-6.PMID 17306674.

9. Gavrilova-Jordan LP, Rose CH, Traynor KD, Brost BC, Gostout BS. Successful term pregnancy following MR-guided focused ultrasound treatment of uterine leiomyoma. J Perinatol 2007; 27:59-61.PMID: 17180132.

10. Funaki K, Sawada K, Maeda F, Nagai S. Subjective effect of magnetic resonance - guided focused ultrasound surgery for uterine fibroids. Journal of Obstetrics and Gynecology Research.2007; 33(6):834-839.PMID:18001451.

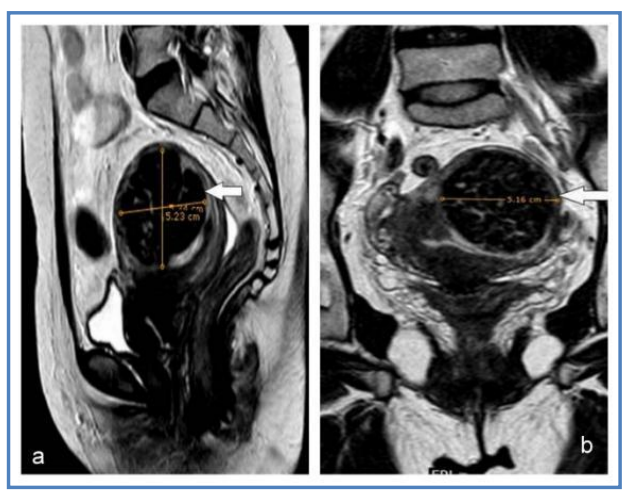

Fig. 1 a \& b: Forty nine year old woman. T2WI sagittal (a) and coronal (b) sequences showing fundal and anterior wall intramural fibroid measuring $5.6 \times 5.1 \times 5.7 \mathrm{~cm}$ (arrows). The fibroid is hypointense to myometrium.
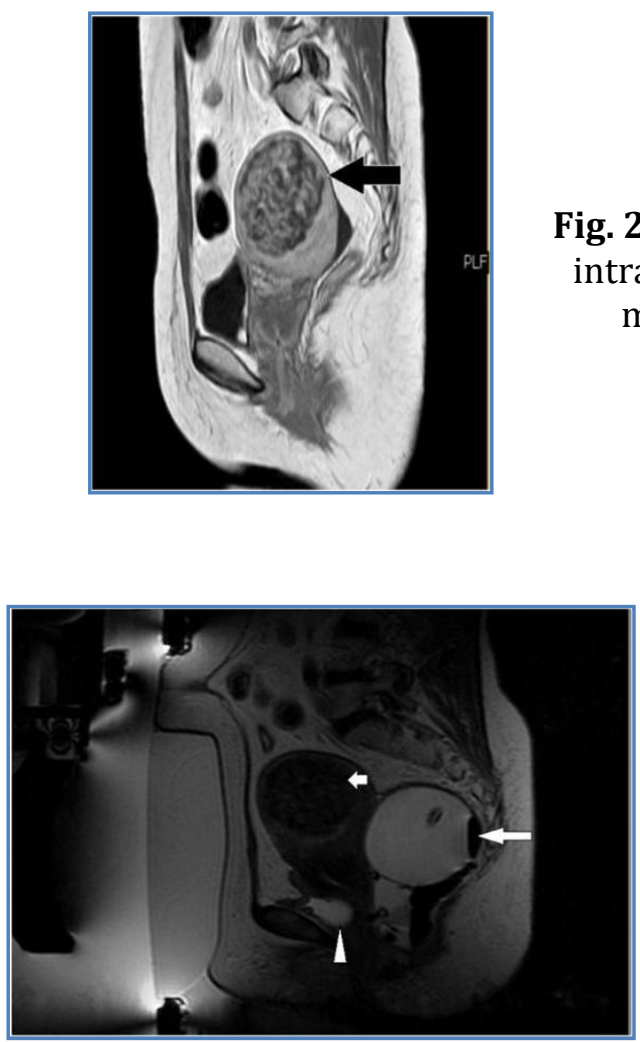

Fig. 2: Forty nine year old woman with a fundal and anterior wall intramural fibroid. Post gadolinium T1WI sagittal images show moderate heterogeneous contrast enhancement (arrow).
Fig. 3: Forty nine year old woman with a fundal and anterior wall intramural fibroid. T2WI sagittal images obtained during treatment planning. The empty urinary bladder with Foley's bulb in situ (arrow head), inflated rectal balloon (long arrow), is seen pushing the fibroid (short arrow) and bringing it close to the anterior abdominal wall. 


\section{CASE REPORT}

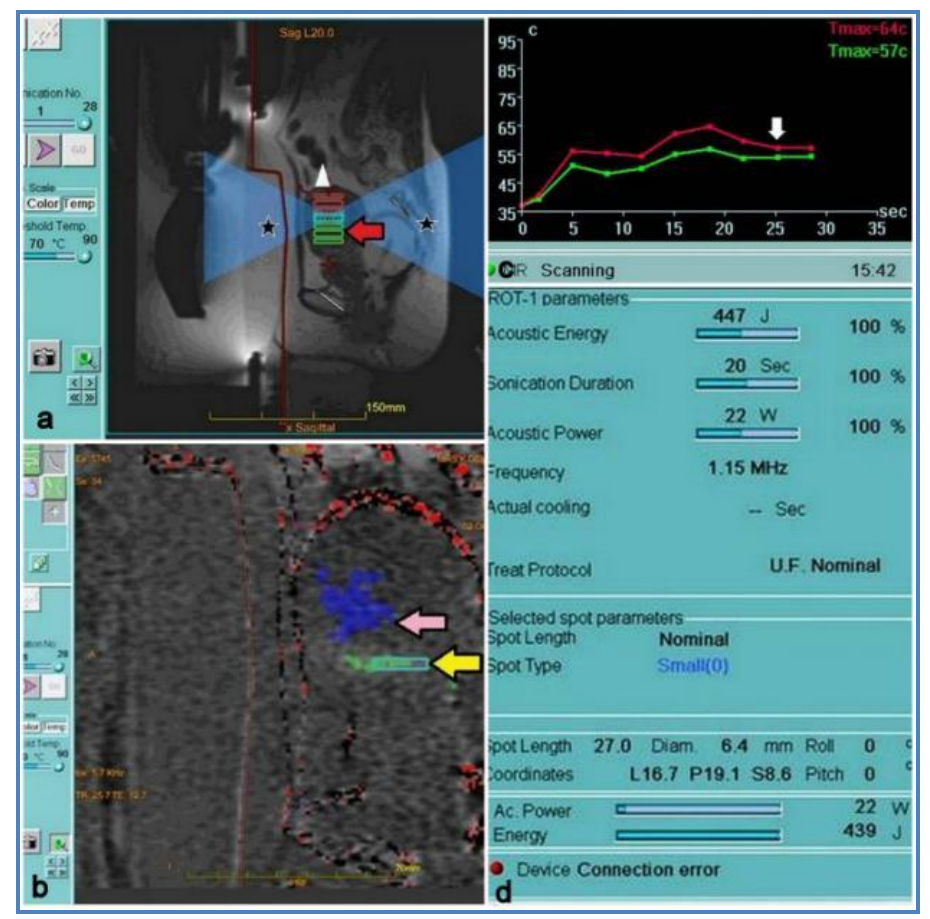

Fig. 4 (a -d): Sagittal (a) T2WI images obtained during treatment show sonication spots (red arrow, ultrasound beam path ( ${ }^{*}$ ) Red line: skin surface; white line: pubic bone; blue line: far field bone; Arrow head: bowel. b post sonication image showing sonicated spot (yellow arrow), previously ablated area (pink arrow) (Protocol: 1.5T).

MRI+ ExAblate 2000, TR: 4200ms, TE: $86.8 \mathrm{~ms}$.cc temperature graph shows the temperature attained at the sonication spot during treatment (arrow). Fig. $d$ the system allows changes to the energy, frequency, spot parameters depending on the patient comfort and the response of the fibroids to the sonications. The system also displays the spot length, diameter of the sonication spot along with the angulations of the beam (roll and pitch).

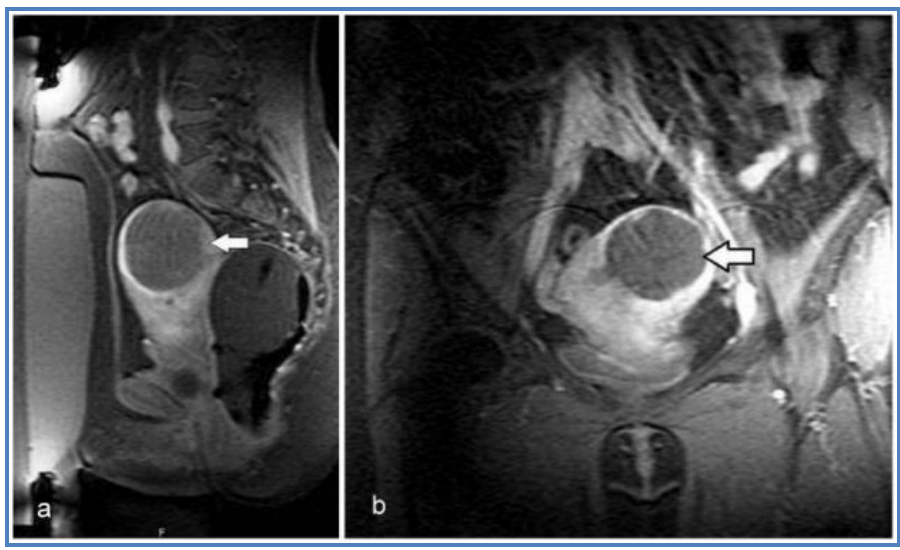

Fig. 5 a \& b: A forty nine year old woman with anterior wall intramural fibroid Contrast enhanced spoiled gradient recalled acquisition in the steady state (SPGR) sagittal (a) and coronal (b)MR of the pelvis after MRgFUS treatment shows non enhancing area (arrows) corresponding to non-perfused volume of $95 \%$. 


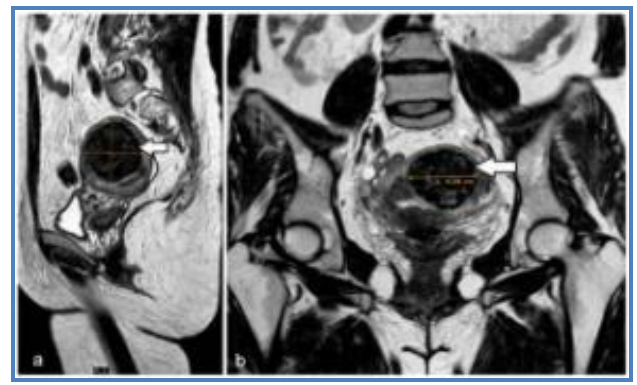

Fig. 6 a \& b: A forty nine year old woman with a fundal and anterior wall intramural fibroid. Sagittal (a) and coronal

(b) T2WI images obtained six months after MRgFUS treatment shows regression of the fibroid in all three planes. The fibroid measures $4.5 \times 4.2 \times 4.7 \mathrm{~cm}$.

\section{AUTHORS:}

1. Anindita Mishra

2. N. Vinay

\section{PARTICULARS OF CONTRIBUTORS:}

1. Professor, Department of Radiodiagnosis, GSL Medical College, Rajahmundry Godavari Imaging Institute, Rajahmundry.

2. Professor \& HOD, Department of Radiodiagnosis, GSL Medical College, Rajahmundry Godavari Imaging Institute, Rajahmundry.

\section{NAME ADDRESS EMAIL ID OF THE}

\section{CORRESPONDING AUTHOR:}

Dr. Anindita Mishra,

Professor,

Department of Radiodiagnosis,

GSL Medical College, Rajahmundry.

Email - dr.aninditamishra@gmail.com

Date of Submission: 30/09/2013.

Date of Peer Review: 01/10/2013.

Date of Acceptance: 05/10/2013.

Date of Publishing: 09/10/2013 